\title{
Liquid In-situ Transmission Electron Tomography Using Hitachi HILEM IL1000 ${ }^{\mathrm{TM}}$ Ionic Liquid.
}

\author{
James P. Kilcrease ${ }^{1}$, Edgar Voelk1 ${ }^{1}$ \\ 1. Hitachi High Technologies America, Inc. Nanotechnology Systems Division, Clarksburg, MD USA
}

Observing delicate biological and/or aqueous material-based samples can require difficult and timeconsuming procedures. Worse, these procedures may involve toxic fixatives, resins, heavy metals, or radioactive materials and ultimately induce changes in the sample's natural state or structure. However, if one were to replace the water in an organic structure by a stable, non-toxic ionic fluid that does not evaporate under vacuum conditions, then sample preparation would become much simpler. HILEM IL $1000^{\mathrm{TM}}$ is an ambient temperature molten organic salt, more commonly known as an 'ionic liquid', designed for specific use in the field of electron microscopy with the goal to preserve structural integrity while avoiding many of the difficulties encountered with traditional preparation techniques [1,3]. In particular, the ionic liquid's choline-like structure offers a biology compatible $\mathrm{pH}$ of 7.4, high ion conductivity, increased stability under vacuum, and a LD 50 of less than $2000 \mathrm{mg} / \mathrm{kg}$ [2,3]. Already, ionic liquids are utilized with increasing success in the field of scanning electron microscopy (SEM), stifling sample charging artifacts, desiccation under high vacuum conditions, and for maintaining the structural integrity of a sample's native conformation [2].

Such properties should make ionic liquids an excellent choice for TEM samples that contain significant amounts of water or even require a liquid environment. While structural preservation is to be expected for the TEM just as for the SEM, it appears liquid environment conditions are possible and sustainable even under TEM tomography conditions.

Here, we chose $\mathrm{Au}$ nanoparticles suspended in water to investigate stability under tomography conditions. Typically, the suspension would be deposited on a copper grid covered with holey carbon and a thin layer of continuous, amorphous carbon. Instead, we mixed a 20\% HILEM IL1000 (diluted in water) concentration 1:1 with the nanoparticle solution. The resulting fluid was then placed directly onto a copper grid with holey carbon only (no support film). Excess fluid was removed with filter paper leaving remaining ionic liquid to form menisci containing nanoparticles in the voids of the holey carbon (see Figure 1). The grids were then imaged at $80 \mathrm{kV}$ in a Hitachi HT7700 TEM with a $\mathrm{LaB}_{6}$ filament and an AMT XR401-B camera. SerialEM was utilized to collect a $\pm 50^{\circ}$ tilt series at $30 \mathrm{kX}$ magnification on individual nanoparticles [4]. 3Dmod / etomo (part of iMOD http://bio3d.colorado.edu/imod/) were used for alignment and viewing of the collected tilt-series. Figure 2 shows the excellent stability, electron transparency and apparent cleanliness of the ionic fluid throughout $\pm 50^{\circ}$ tilt range. No detectable movement within the fluid was found throughout the series, even for the Au gold particle itself at high tilt angles.

The advantages of ionic liquids for TEM analyses of aqueous material and biological samples such as virus, bacteria, and proteins are significant. Structural investigations and native state conformations become easier with Hitachi's ionic liquid HILEM IL $1000^{\mathrm{TM}}$ that is above all non-toxic with a biologically equivalent $\mathrm{pH}$ of 7.4 and even maintains the ability to conduct tomography investigations in a fluid environment. 


\section{References:}

[1] Shiono et al. Microsc. Microanal.20, Suppl 3 (2014), p. 1016.

[2] Kawai, K., et al. Langmuir Letters 27 (2011) p. 9671-9675.

[3] Sakaue et al. Microsc. Microanal.20, Suppl 3 (2014), p. 1012.

[4] The authors acknowledge Dr. Shouzhong Zou for allowing us to utilize the gold nanoparticles.
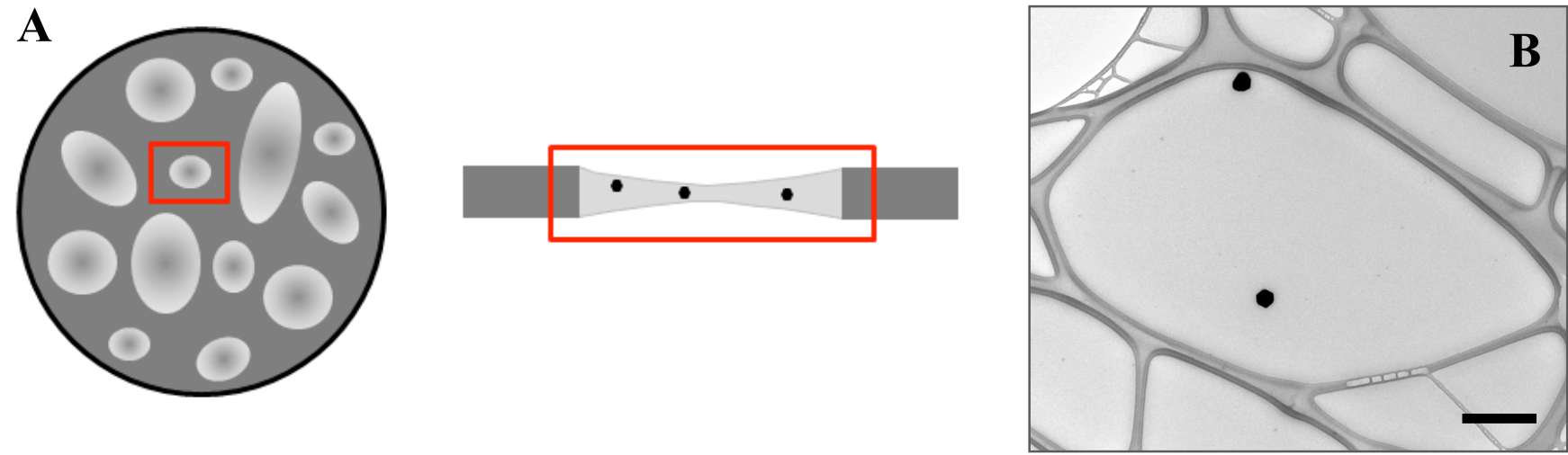

Figure 1. HILEM IL1000 as liquid in-situ suspension for nanoparticles. Figure A; diagram of a copper grid with holey carbon and no support film utilizing ionic liquid as a suspension medium filling in voids. Middle image demonstrates how a meniscus of ionic liquid is formed containing a sample of interest (nanoparticles). Panel B demonstrates gold nanoparticles suspended in ionic liquid with the far top left hole (white) showing an area where the ionic liquid meniscus has broken. Scale bar; 500nm.
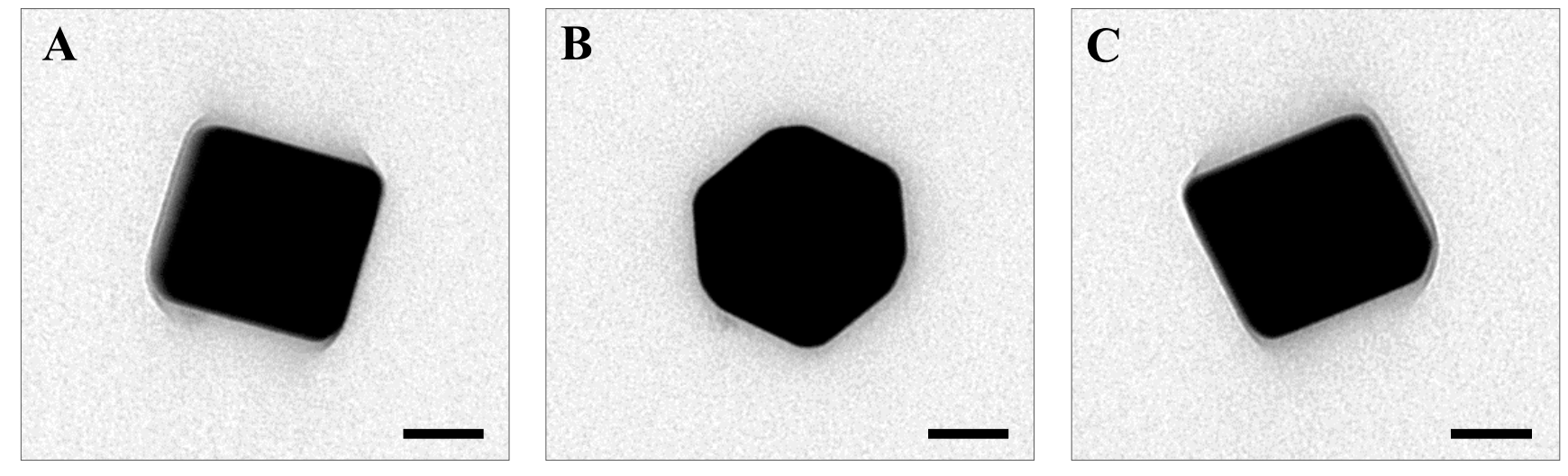

Figure 2. Gold octahedral nanoparticle approximately $130 \mathrm{~nm}$ in diameter (point to point) suspended in ionic liquid on copper grid with holey carbon during 100 degree tilt series. Panel A demonstrates tilt orientation at -50 degrees, panel B at 0 degrees, and panel C at +50 degrees. Scale bars; $50 \mathrm{~nm}$. 